\title{
Práticas integrativas e complementares em saúde: possibilidades para o cuidado integral
}

\author{
Integrative and complementary practices in health: possibilities for integral care
}

Prácticas integrativas y complementarias en salud: posibilidades de atención integral

Phillipe Augusto Marques Silva ${ }^{1 *}$, Aline Emanuele Ferreira Oliveira1', Bianca Emanuelle de Souza1, Cecília Tavares Barbosa ${ }^{1}$, Letícia Silva Oliveira ${ }^{1}$, Samira Auxiliadora Pereira', Natália de Cássia Horta ${ }^{1}$.

\section{RESUMO}

Objetivo: Apresentar o processo de construção e implementação do plano municipal de Práticas Integrativas e Complementares em Saúde (PICS), por meio do mapeamento do conhecimento e da demanda dos profissionais e usuários da rede assistencial de saúde. Métodos: Trata-se de uma pesquisa-ação, desenvolvida em 2019, em um município de Minas Gerais, por meio da construção de um diagnóstico situacional após aplicação de questionários para usuários, agentes comunitários de saúde e profissionais de saúde das demais categorias, atuantes principalmente na Atenção Primária à Saúde. O estudo foi aprovado por Comitê de Ética em Pesquisa. Resultados: Constatou-se que parcela significativa dos usuários faz o uso regular de pelo menos uma das PICS e gostariam de utilizar tais práticas de modo complementar à terapia tradicional, caso sejam indicadas por um profissional de saúde. Contudo, embora a maioria dos profissionais do município tenha interesse em implementar as práticas no cuidado diário dos seus pacientes, a oferta dessas práticas ocorre de maneira não sistematizada, sem amparo da Gestão Municipal. Conclusão: 0 cenário mapeado indica a necessidade de intervenções que favoreçam a construção e consolidação sistematizada das PICS de modo participativo, como uma proposição de política municipal de saúde, com participação ativa de usuários, profissionais de saúde, e instituições educacionais.

Palavras-chave: Terapias complementares, Atenção primária à saúde, Saúde pública.

\begin{abstract}
Objective: To present the process of construction and implementation of the municipal plan of Integrative and Complementary Health Practices (PICS), through the mapping of knowledge and demand from professionals and users of the health care network. Methods: This is an action-research, developed in 2019 in a city of Minas Gerais, through the construction of a situational diagnosis after applying questionnaires to users, community health agents and health professionals from other categories, mainly active in Primary Health Care.The study was approved by the Research Ethics Committee. Results: It was found that a significant portion of users make regular use of at least one of the PICS and would like to use such practices in a complementary manner to traditional therapy, if indicated by a health professional. However, although most professionals in the municipality are interested in implementing the practices in the daily care of their patients, the offer of these practices occurs in an unsystematized manner, without support from the Municipal Management. Conclusion: The mapped scenario indicates the need for interventions that favor the construction and systematic consolidation of PICS in a participatory manner, as a proposal of municipal health policy, with active participation of users, health professionals, and educational institutions.
\end{abstract}

Keywords: Complementary therapies, Primary health care, Public health.

1 Pontifícia Universidade Católica de Minas Gerais (PUC Minas), Betim - MG.

*E-mail: phillipe.marques2@gmail.com 


\section{RESUMEN}

Objetivo: Presentar el proceso de construcción e implementación del plan municipal de Prácticas Sanitarias Integradoras y Complementarias (PICS), a través del mapeo del conocimiento y la demanda de los profesionales y usuarios de la red de salud. Métodos: Se trata de una investigación de acción, desarrollada en 2019 en una ciudad de Minas Gerais, mediante la construcción de un diagnóstico situacional tras aplicar cuestionarios a los usuarios, los agentes sanitarios de la comunidad y los profesionales de la salud de las demás categorías, que trabajan principalmente en la Atención Primaria de Salud.El estudio fue aprobado por el Comité de Ética en Investigación. Resultados: Se comprobó que una parte importante de los usuarios utilizan regularmente por lo menos una de las PICS y desearían utilizar esas prácticas de manera complementaria a la terapia tradicional, si así lo indica un profesional de la salud. Sin embargo, aunque la mayoría de los profesionales del municipio están interesados en aplicar las prácticas en el cuidado diario de sus pacientes, la prestación de estas prácticas se produce de manera no sistematizada, sin el apoyo de la Dirección Municipal. Conclusión: El escenario mapeado indica la necesidad de intervenciones que favorezcan la construcción y consolidación sistemática de la PICS de manera participativa, como propuesta de política municipal de salud, con la participación activa de los usuarios, profesionales de la salud e instituciones educativas.

Palabras clave: Terapias complementarias, Atención primaria de salud, Salud pública.

\section{INTRODUÇÃO}

As Práticas Integrativas e Complementares em Saúde (PICS) são métodos diagnósticos e terapêuticos, advindos principalmente da Medicina Oriental, que têm como objetivo estimular os mecanismos naturais e intrínsecos do organismo no processo saúde-doença-cuidado (BRASIL, 2018).

A partir de uma visão ampliada do ser humano, do meio ambiente e da sociedade, as PICS pautam-se na escuta acolhedora, desenvolvimento do vínculo terapêutico e estímulo ao autocuidado. São também denominadas como Medicina Tradicional e Complementar/Alternativa (MT/MCA) pela Organização Mundial da Saúde (OMS) (WORLD HEALTH ORGANIZATION, 2002).

O Ministério da Saúde (MS), considerando as orientações da OMS e a demandas levantadas durante as conferências nacionais, lançou em 2006 a Política Nacional de Práticas Integrativas e Complementares (PNPIC) no Sistema Único de Saúde (SUS), por meio da Portaria n 971/2006. 29 práticas são contempladas, dentre elas: plantas medicinais, fitoterapia, medicina tradicional chinesa/acupuntura.

A PNPIC proporciona e viabiliza a articulação das PICS com a Rede de Atenção à Saúde (RAS), uma vez que norteia a federação, os estados e os municípios quanto aspectos técnicos, financeiros e científicos do processo de implementação das práticas complementares, respeitando as particularidades culturais e socioeconômicas de cada região (BRASIL, 2015; WORLD HEALTH ORGANIZATION, 2002). Desse modo, as PICS representam uma importante ferramenta de ampliação e efetivação das políticas de saúde ao demonstrarem diversos benefícios do ponto de vista da integralidade.

O objetivo do presente estudo é elaborar um plano de implantação das PICS, em um município do estado de Minas Gerais, a partir de um diagnóstico situacional acerca do uso dessas prática pela população, haja vista a não implementação da PNPIC pelo município, tendo em vista a demanda das PICS pelos usuários e profissionais e pelo uso de algumas dessas práticas, por iniciativas isoladas de profissionais, mesmo sem protocolos ou apoio da Gestão Municipal.

\section{MÉTODOS}

Trata-se de pesquisa-ação, de cunho qualitativo, desenvolvida no primeiro semestre de 2019. Tal escolha metodológica propõe aproximar da realidade social, das crenças e das simbologias da vida, visando o conhecimento científico e a transformação da realidade. 
A pesquisa-ação como uma abordagem metodológica é um tipo de pesquisa social que permite compreender uma realidade, considerando o seu contexto coletivo; identificar seus desafios e intervir, a partir de proposição aos problemas evidenciados, permitindo que pesquisadores e participantes interajam de forma colaborativa e participativa (THIOLLENT M, 2017). Esta pesquisa-ação fez parte do Internato Obrigatório em Saúde Coletiva (ISC) do curso de Medicina de uma universidade de Minas Gerais.

$\mathrm{Na}$ primeira etapa construiu-se o diagnóstico situacional, aplicando questionários aos participantes. Concomitantemente, os internos do Curso de Medicina e alguns representantes da Secretaria Municipal de Saúde do município mineiro criaram a comissão de implantação das PICS na cidade, após mapeamento e convite de profissionais-chave (BRASIL, 2018). Foram 10 integrantes, dentre eles: médico, enfermeira, farmacêuticos, educador físico, fisioterapeuta, psicóloga e engenheiro agrônomo.

A pesquisa teve como cenário as 23 UBS, as 5 equipes do NASF e o Centro de Especialidades Médicas Municipal. Foram convidados a participar todos os profissionais de saúde atuantes nestes serviços: médicos, enfermeiros, técnicos de enfermagem, agentes comunitários de saúde (ACS), agentes de combate a endemias, farmacêuticos, técnicos farmacêuticos, assistentes sociais, terapeutas ocupacionais, psicólogos, nutricionistas, educadores físicos, fisioterapeutas e dentistas; bem como alguns usuários das UBS. O questionário dos ACS foi individualizado em relação aos demais profissionais, visando observar o perfil como usuários e profissionais, já que estão inseridos no território e mais próximos da população.

Os questionários foram desenvolvidos pelos pesquisadores e contemplaram a identificação dos participantes, o seu conhecimento e o uso pessoal das PICS. Aos profissionais, também foi perguntado sobre prescrição, formação/capacitação e o interesse em PICS, além da indicação de profissionais do município que possuem formação nestas práticas. O estudo foi submetido ao Comitê de Ética e Pesquisa (CEP), em conformidade com a Resolução 466/12, com parecer de aprovação n. 3.452.609/19.

Ao todo, foram respondidos 436 questionários, sendo: 190 pelos usuários, 132 pelos ACS e 114 profissionais de saúde das demais categorias da rede assistencial. Todos os participantes assinaram o Termo de Consentimento Livre e Esclarecido (TCLE).

Os questionários foram tabulados em planilhas do Microsoft Excel®, obtendo os dados quantitativos para as questões polares (que continham opções "sim" e "não") e analisando o conteúdo das questões abertas a partir de categorias definidas. Desta forma, 4 categorias analíticas foram construídas: perfil dos participantes e formação em PICS; conhecimento e uso das PICS; aspectos facilitadores e desafios para implantação das PICS e perspectivas e proposições de ações para PICS no município do interior de Minas Gerais.

\section{RESULTADOS E DISCUSSÃO}

\section{Perfil dos Participantes e Formação em PICS}

436 indivíduos responderam os questionários sendo 190 (43,6\%) usuários, 132 (30,3\%) ACS e 114 $(26,1 \%)$ demais profissionais da rede. A maior parte dos entrevistados foram mulheres $(83,5 \%)$, refletindo a preponderância do sexo feminino nos contextos de cuidado e assistência. A faixa etária média foi entre 31 e 40 anos e a maioria discreta $(55,7 \%)$ são casados.

Sobre a escolaridade, apenas $26,3 \%$ dos usuários possuíam ensino médio completo, já $82,2 \%$ dos ACS alegaram ter o mesmo grau de escolaridade. Entre os demais profissionais da saúde, $75,5 \%$ concluíram o ensino superior e $36,6 \%$ possuíam pós-graduação.

$\mathrm{Na}$ análise do vínculo de trabalho, especificamente aos usuários, a maioria dos entrevistados estavam empregados formalmente $(54,7 \%)$, seguido pela ocupação de dona de casa/do lar (25,2\%). No grupo dos demais profissionais da saúde, houve preponderância exatamente dos cargos essenciais à ESF: $28 \%$ médicos(as), $25 \%$ enfermeiros(as), $24 \%$ técnicos(as) de enfermagem e $23 \%$ outras categorias profissionais (educadores físicos, farmacêuticos, nutricionistas, psicólogos, entre outros).

Sobre formação em PICS, 90,9\% dos ACS e $91,2 \%$ dos demais profissionais disseram não ter nenhuma formação em PICS. Dos profissionais que possuem formação, obteve-se: 2 em auriculoterapia, 1 em plantas medicinais (PM), 1 em yoga, 1 em medicina tradicional chinesa (MTC) e 1 em meditação. Dos ACS que alegaram possuir formação nas práticas, apenas 1 participou de um curso de meditação. 
O presente estudo também analisou o conhecimento dos profissionais sobre a oferta de cursos de formação e aperfeiçoamento em PICS pelo MS. Dos participantes, $75,4 \%$ dos profissionais alegaram desconhecimento. Entre os ACS, essa porcentagem é ainda maior (96,1\%).

Dos profissionais que afirmaram conhecer os cursos, $10,7 \%$ já realizaram algum, sendo os mais citados: auriculoterapia, PM e MTC. Entre os ACS, nenhum entrevistado havia realizado os cursos, mas $26 \%$ demonstraram interesse, principalmente em fitoterapia, yoga e MTC.

Em Florianópolis-SC, Thiago SCS e Tesser CD (2011) analisaram a percepção dos profissionais da ESF sobre as PICS, sendo que os resultados apresentados se assemelham ao estudo proposto: $87,6 \%$ dos profissionais não possuíam especialização em PICS; 88,7\% dos participantes desconheciam as diretrizes nacionais para a área; e 59,9\% demonstraram interesse em capacitar-se em algumas das práticas (THIAGO SCS e TESSER CD, 2011).

No que se refere a prescrição das PICS, apenas 33,3\% dos profissionais afirmaram prescrever alguma das práticas nos cuidados para com seus pacientes. Em contrapartida, $62,1 \%$ dos ACS e $78,8 \%$ dos usuários declaram que gostariam de utilizar alguma PM, de modo complementar à terapia alopática, caso fosse recomendada por um profissional de saúde.

Já Mattos G et al. (2018) analisaram o uso das PM e fitoterápicos na atenção primária por médicos, enfermeiros e auxiliares de enfermagem. $84,7 \%$ dos profissionais entrevistados já prescreveram e/ou sugeriram o uso das PICS aos seus pacientes e 70,7\% indicaram que a população demonstra interesse nessas práticas (MATTOS G, et al., 2018).

A maioria dos profissionais entrevistados (71\%) alegaram não se sentirem aptos a incluir as PICS na sua rotina profissional e $84,2 \%$ não tem o costume de perguntar aos usuários sobre o uso dessas práticas. Contudo, 45,6\% afirmaram que gostariam de aprofundar seus conhecimentos em PICS e aplicá-los em sua prática clínica.

As práticas de maior interesse foram: yoga, auriculoterapia, homeopatia, MTC e meditação. Esses achados reforçam o interesse dos participantes pelo aprofundamento dessas práticas e a importância de que, dentro das proposições do plano municipal das PICS na cidade, exista um eixo de educação permanente, visando a qualificação dos profissionais da APS.

\section{Conhecimento e Uso das PICS}

Com relação ao conhecimento acerca das PICS, $68,8 \%$ dos usuários entrevistados disseram não conhecer as práticas integrativas. Dentre os que conhecem (31,1\%), as mais citadas foram as PM $(36,2 \%)$, a MTC $(29,3 \%)$ representada, principalmente, pela acupuntura e pela auriculoterapia e a yoga $(10,3 \%)$. Por outro lado, $74,2 \%$ dos ACS afirmaram conhecer uma ou mais modalidades de PICS, sendo as mais lembradas as PM $(77,5 \%)$, seguidas por yoga $(59,1 \%)$, meditação $(46,9 \%)$, homeopatia $(39,7 \%)$ e MTC $(32,5 \%)$. Essa alta porcentagem também foi vista no grupo dos demais profissionais da saúde, já que $72,8 \%$ conhecem, pelo menos, uma prática integrativa. Nesse grupo, as mais mencionadas foram a homeopatia $(50,9 \%)$, a yoga $(50 \%)$ e as PM $(47,3 \%)$.

Chama a atenção que a maioria dos usuários entrevistados afirmam não conhecer nenhuma modalidade de PICS e, principalmente, que não consideram e/ou reconhecem as PM como exemplo de tais práticas. $O$ que corrobora com 0 fato de as terapias oriundas da MT/MCA terem sido subvalorizadas no país, historicamente, como consequência do modelo biomédico de atenção à saúde vigente (LIMA CA, 2018; PASSOS MMB, 2018).

Esse paradigma é caracterizado por uma assistência fragmentada, mecanicista, curativista e hospitalocêntrica que se contrapõe ao modelo de determinação social da doença. Este reconhece a importância de conceitos norteadores da saúde pública, da saúde mental e das ciências sociais para a caracterização do conceito de "saúde", além de posicionar o indivíduo no centro do processo saúde-doençacuidado (SOUSA IV, et al., 2019). Nesse contexto, conclui-se que as PICS são um importante instrumento para a mudança do modelo assistencial que estamos vivendo, ao propor uma nova compreensão do processo de cuidado humano, especialmente do autocuidado (SOUSA IV, et al., 2019; HARAGUCHI LMM, 2020). 
É inquestionável a relação direta entre as variáveis socioeconômicas (sexo, idade, escolaridade e renda familiar) e o acesso e o uso das práticas integrativas (LIMA CA, 2018). Quanto maior o nível de escolaridade, maiores as taxas de conhecimento e de uso de, ao menos, uma modalidade de prática integrativa, fenômeno observado no grupo dos ACS e dos demais profissionais da saúde. Similarmente ao que foi encontrado nesse estudo, Lima CA et al. (2018) constataram as seguintes associações estatisticamente significantes: homeopatia e ensino superior, quiropraxia e renda superior ou igual a quatro salários mínimos.

Considerando a importância histórica e cultural do cultivo de PM no Brasil, especialmente em hortas e pomares familiares, já era de se esperar que essa modalidade de PICS figurasse entre as mais citadas em todos os grupos pesquisados no estudo neste município mineiro também reconhecido pelo cultivo de hortaliças (LIMA CA, 2018).

Estudos semelhantes conduzidos em outros municípios brasileiros também evidenciaram maiores taxas de reconhecimento e uso das PM enquanto terapia complementar, sobretudo pela população em geral (OLIVEIRA DMS e LUCENA EMP, 2015; MESSIAS MCTB, et al., 2015; BADKE MR, et al., 2019; FLOR ASSO e BARBOSA WLR, 2015).

Apesar da maioria dos ACS conhecerem as PICS, 55,8\% disseram não fazer o uso pessoal de nenhum tipo de terapia alternativa e complementar. Dos adeptos (44,1\%), as PM foram mencionadas por todos os entrevistados. O mesmo pode ser notado dentre os outros profissionais, já que apenas $32,4 \%$ deles utilizam alguma PICS, sendo que as práticas mais citadas foram as PM $(37,8 \%)$ e a homeopatia $(21,6 \%)$.

O amplo uso das PM, especialmente pela população de baixa renda, pode ser explicada pela popularidade dessa prática no Brasil pelas origens históricas, baixo custo e pelo fácil acesso às plantas (LIMA CA, 2018; BADKE MR, et al., 2019; CHIBANTE CLP, et al., 2017). Por isso, neste estudo, optou-se por investigar mais a fundo o conhecimento e as formas de uso das PM e dos fitoterápicos em ambos os grupos de usuários e ACS.

No que tange ao conhecimento e/ou uso das PM, $55 \%$ dos usuários pesquisados fazem seu utilizam sem as reconhecer como PICS. Dentre os ACS, $70,4 \%$ relataram utilizar rotineiramente tais plantas. Em ambos os grupos, as mais referidas foram a erva cidreira, a hortelã, o boldo, o alecrim, a folha de algodão e a tanchagem. Os objetivos do uso dessas plantas mais citados foram o tratamento de afecções respiratórias, infecções urinárias, situações de estresse e ansiedade.

emelhante ao que encontramos, Oliveira VB et al. (2018) identificaram que $72,2 \%$ dos usuários de uma UBS em Colombo/PR utilizam PM, visando principalmente os efeitos calmantes e o tratamento de afecções estomacais. É importante mencionar que houve discordância entre as plantas mais mencionadas neste estudo, em comparação com trabalhos conduzidos em outras regiões do país, o que remete à vasta biodiversidade brasileira, assim como os distintos nomes populares para as mesmas espécies (OLIVEIRA VB, et al., 2018; COLET CF, et al., 2015).

Quanto ao local de aquisição das PM pelos usuários, as hortas de casa (47\%) foram as mais apontadas, seguido pela obtenção por meio de vizinhos $(33,7 \%)$ e casas de plantas ou raizeiros $(8,7 \%)$. A aquisição residencial é apontada em diversos estudos, evidenciando o aspecto familiar de transmissão de informações acerca da forma de uso e indicação das PM, repassadas de geração à geração (OLIVEIRA VB,et al., 2018; SILVA TC, et al., 2018; LARA AC, et al., 2019).

Esse aspecto afeta, inclusive, o próprio conhecimento e prescrição entre profissionais, que por vezes tiveram esse primeiro contato e ensino oriundos do ambiente familiar, mesmo antes da possível formação acadêmica sobre o tema (MATTOS G, et al., 2018; SCHIAVO M, et al., 2017).

As formas de maior utilização das PM pelos ACS foram: chá $(72 \%)$, pomada $(11,3 \%)$, imersão $(8,6 \%)$ e óleo (6\%). A simplicidade no modo de preparo dos chás foi um dos motivos apontados na literatura para justificar a preponderância nessa forma de utilização (COLET CF, et al., 2015; LARA AC, et al., 2019). É importante ressaltar que nem sempre os usuários estão informados sobre o correto preparo das PM para obter os efeitos terapêuticos. Orientação adequada por parte de profissionais é necessária,visando evitar intoxicações, interações medicamentosas indesejadas e efeitos adversos (LARA AC, et al., 2019). 
Nesse contexto, o ACS se destaca como peça fundamental no fortalecimento e implementação da ESF no SUS, uma vez que a característica da profissão é estar junto das famílias, inseridos na comunidade, sendo muitas vezes fonte de informação e esclarecimentos aos usuários, o que reforça ainda a necessidade de capacitação deste grupo (SCHIAVO M, et al., 2017).

Sobre o conhecimento e/ou uso de fitoterápicos,aproximadamente $79 \%$ dos usuários não fazem ou nunca fizeram o uso de medicamentos fitoterápicos. Esses achados se repetiram entre os ACS, já que $78 \%$ referiram nunca ter feito o uso de nenhuma dessas medicações. Entre aqueles que os usam, as medicações fitoterápicas mais lembradas entre os dois grupos de entrevistados foram os calmantes à base de Passiflora incarnata e do extrato de própolis.

No município mineiro, através da vivência do ISC e dos indicadores de saúde municipal, notou-se uma elevada dispensação de medicamentos para transtornos de ansiedade e distúrbios do sono. Assim, os fitoterápicos apresentam uma ampla aplicabilidade, com comprovação científica, sendo alternativa a alguns medicamentos alopáticos. Silva TC et al. (2018) apontam para o fato das medicações calmantes, como a Pasalix $\AA^{\circledR}$ (a base de Passiflora incarnate) estarem entre as mais procuradas numa farmácia comunitária no município de Cascável/CE.

Neste estudo, essa espécie foi a mais citada por usuários e ACS como fitoterápicos conhecidos. Esses achados corroboram para necessidade de implementação da PMPIC nesta cidade, principalmente no que tange o investimento em serviço sistematizado de cultivo, preparo e dispensação de fitoterápicos ao moldes de outros municípios que já possuem o programa da Farmácia Viva (COLET CF, et al., 2015; SILVA LAD e ALBIERO ALM, 2014; SILVA MIG, et al., 2006).

\section{Aspectos Facilitadores e Desafios para a Implementação das PICS}

O diagnóstico das PICS evidenciou que os usuários e os ACS conhecem e demostram interesse no uso das PM e fitoterápicos. Boccolini PMM e Boccolini CS (2020) realizaram um estudo com os dados da Pesquisa Nacional de Saúde de 2013 e encontram que as práticas mais utilizadas no Brasil foram as PM e a fitoterapia.

Eles também viram que um dos fatores influenciadores desse achado foi o baixo custo dessas terapias e o caráter sociocultural dessas práticas (BOCCOLINI PMM, BOCCOLINI CS, 2020). Na cidade de Minas Gerais em que a pesquisa ação foi construída não foi diferente, já que há uma forte presença das hortas familiares e do cultivo de PM que favorecem ainda mais a implementação no município, como já mencionado.

Quando questionados sobre os pontos facilitadores para a aplicação das PICS em seu exercício diário, os profissionais da saúde concordam que o interesse dos usuários às práticas é um benefício, contribuindo para adesão.

Em relação a aceitação do uso de PICS pelos profissionais, Ruela LO, et al. (2019) diz que a pouca adesão dos profissionais se deve a baixa comprovação científica. No entanto, no presente estudo, encontrou-se profissionais com um grande envolvimento, alguns até ministrando algumas PICS por iniciativa própria e empenhados com a criação da comissão para avançar nas discussões sobre o assunto.

Porém ainda existem obstáculos para sua efetiva implantação no município. Quando abordados sobre os maiores empecilhos à aplicação das PICS na sua prática, os profissionais de saúde alegaram falta de conhecimento, alto número de atendimentos diários e espaço físico inadequado. Soma-se a isso o fato de que o município também não tinha um Plano Municipal de Educação Permanente (PMEP) que teve sua construção iniciada pelo ISC, mas que não contemplou as terapias complementares. Isso dificulta a formação e qualificação dos profissionais inseridos na rede, mesmo que esses recursos humanos sejam fundamentais na implementação da PNPIC (RUELA LO, et al., 2019).

O apoio da gestão também é essencial para a inserção das práticas e, nesse sentido, desde o início, a gestão da Secretária de Saúde do município mineiro se interessou na proposta, inclusive tendo representantes na comissão (RUELA LO, et al., 2019; MATTOS G, et al., 2018).

Embora exista o suporte da gestão, alguns impasses permanecem, principalmente em relação ao repasse financeiro pelo Estado e Federação para realização e implementação das PICS, já que é necessário o investimento em insumos e na educação permanente dos profissionais (TESSER CD, et al., 2018). 


\section{Perspectivas e Proposições de Ações para Implantação das PICS}

A partir do diagnóstico situacional, várias ações foram implementadas no contexto das PICS no município da intervenção, em Minas Gerais. Durante o 1ำ semestre de 2019, como primeira etapa de institucionalização da PICS no município, foi criada comissão. Na comissão, há uma representante da Secretaria de Saúde da cidade, o que favorece a implementação dos processos definidos pelo grupo, junto do Plano Municipal de Saúde.

Os internos, junto à comissão, discutiram as etapas propostas pelo Manual de Implantação de Serviços de PICS no SUS. Duas fases foram mais exploradas: definição de propostas e elaboração do Plano Municipal de PICS. Essas ações direcionaram os envolvidos, tanto sobre a importância de ter o Plano Municipal formatado a fim de oficializá-lo junto aos editais públicos de financiamento estadual e federal, bem como para divulgá-lo enquanto uma política pública municipal.

No $2^{\circ}$ semestre de 2019 houve inclusão das PICS em processos de capacitação e qualificação dos profissionais, bem como $01^{\circ}$ Seminário sobre as PICS na cidade da pesquisa-ação, com o objetivo de sensibilizar os trabalhadores da saúde sobre o diagnóstico situacional das PICS no município e sinalizar as possibilidades de intervenções com foco nas PM.

Dando continuidade ao diagnóstico situacional, tornou-se fundamental a discussão sobre o manejo e cultivo das PM nas UBS, considerando o investimento na fitoterapia como uma das PICS de maior aproximação e aceitação pelos profissionais e usuários, conforme revelado no diagnóstico.

Neste momento, 14 UBS foram abordadas, e identificou-se 9 com possibilidade de implantação de hortas locais para cultivo e disponibilização de algumas PM. Durante o primeiro semestre de 2020, foi elaborada cartilha sobre as 16 PM mais utilizadas.

Esta intervenção consiste numa parceria da comissão municipal com o ISC. A cartilha traz especificação da planta medicinal (nome científico e nome popular), indicações, forma de preparo e uso. Como próximas etapas, existe a proposta de realização do $2^{0}$ seminário municipal abordando o manejo clínico dos fitoterápicos, além de um curso de auriculoterapia promovido pela Gestão Municipal em parceria com a empresa QualiPhi-Práticas Integrativas. Entretanto, devido à pandemia da COVID-19, tais atividades foram temporariamente suspensas.

\section{CONCLUSÃO}

A partir do diagnóstico situacional, constatou-se que uma parcela significativa dos usuários da APS faz uso regular de pelo menos uma das PICS e gostariam de utilizar tais práticas de modo complementar à terapia tradicional, se indicado por um profissional de saúde. Contudo, embora a maioria dos profissionais do município tenha interesse em implementar as práticas no cuidado diário dos seus pacientes, a oferta dessas práticas ocorre de maneira não sistematizada, sem amparo da Gestão Municipal. Este cenário indica a necessidade de intervenções que favoreçam a construção e consolidação sistematizada das PICS de modo participativo, como uma proposição de política municipal de saúde, com participação ativa de usuários, profissionais de saúde, e instituições educacionais.

\section{REFERÊNCIAS}

1. BADKE MR, et al. Meaningsofthe use of medical plants in self-carepractices. Rev Esc Enferm USP, 2019; 53:1-8.

2. BOCCOLINI PMM, BOCCOLINI CS. Prevalenceofcomplementaryandalternative medicine (CAM) use in Brazil. BMC $\begin{array}{llllll}\text { Complement } \quad \text { Med } & \text { Ther, } 2020 ; & \text { (51). }\end{array}$ https://bmccomplemeNtmedtherapies.biomedcentral.com/articles/10.1186/s12906-020-2842-8.

3. BRASIL. Ministério da Saúde (MS). Manual de implantação de serviços de práticas integrativas e complementares no SUS. Brasília: MS; $2018 . \quad$ Disponível em: http://189.28.128.100/dab/docs/portaldab/publicacoes/manual_implantacao_servicos_pics.pdf

4. BRASIL. Ministério da Saúde (MS). Política nacional de práticas integrativas e complementares no SUS: atitude de ampliação de acesso [documento da Internet]. Brasília: MS; 2015. Disponível em: https://bvsms.saude.gov.br/bvs/publicacoes/pnpic.pdf.

5. CHIBANTE CLP, et al. Knowledgeandpractices in carefocusedonindividualswithwounds. Esc Anna Nery, 201721 (2),1-8. Availablefrom: https://www.scielo.br/scielo.php?pid=S141481452017000200208\&script=sci_arttext\&tIng=en. 
6. COLET CF, et al. Uso de plantas medicinais por usuários do serviço público de saúde do município de ljuí/RS. Ver Bras Med Fam Comunidade, 2015; 10(36):1-13. Disponível em: https://rbmfc.org.br/rbmfc/article/view/930/728

7. FLOR ASSO, BARBOSA WLR. Sabedoria popular no uso de plantas medicinais pelos moradores do bairro do sossego no distrito de Marudá-PA. RevBrasPIMed, 2015;17(4):757-768.

8. HARAGUCHI LMM, et al. Impactofthe training ofprofessionalsfrom São Paulo publichealth system in phytotherapypractice. RevBrasEducMed, 2020; 44(1): 1-11.

9. IBIRITÉ. Secretária Municipal de Saúde. Plano Municipal de saúde 2018-2021. 2017. Documento interno.

10. LARA AC, et al. Avaliação do consumo de produtos naturais por usuários de estratégias de saúde da família do município de Rondonópolis - MT. Revista UniVap, 2019; 25(47):98-109. Disponível em: https://revista.univap.br/index.php/revistaunivap/article/view/2071/1532.

11. LIMA CA, et al. Integrativeandcomplementarypractices: use by Community healthagents in self-care. Rev Bras Enferm, 2018; 71 (Supl. 6): 2682-2688.

12. MATTOS G, et al. Plantas medicinais e fitoterápicos na Atenção Primária em Saúde: percepção dos profissionais. CienSaudeColet, 2018; 23(11):3735-3744.

13. MESSIAS MCTB, et al. Uso popular de plantas medicinais e perfil socioeconômico dos usuários: um estudo em área urbana em Ouro Preto, MG, Brasil. RevBrasPIMed, 2015;17(1):76-104.

14. OLIVEIRA DMS, LUCENA EMP. O uso de plantas medicinais por moradores de Quixadá-Ceará. RevBrasPIMed, 2015;17(3): 407-412.

15. OLIVEIRA VB, et al. Conhecimento e Uso de Plantas Medicinais por Usuários de Unidades Básicas de Saúde na Região de Colombo, PR. R BrasCi Saúde, 2018; 22(1):57-64. Disponível em: http://docs.bvsalud.org/biblioref/2018/03/880718/conhecimento-e-uso-de-plantas-medicinaispo.pdf

16. PASSOS MMB, et al. A disseminação cultural das garrafadas no Brasil: um paralelo entre medicina popular e legislação sanitária. Saúde debate, 2018; 42(116): 248-262.

17. RUELA LO, et al. Implementação, acesso e uso das práticas integrativas e complementares no Sistema Único de Saúde: revisão da literatura. CiencSaudeColet, 2019; 24(11): 4239-4250.

18. SCHIAVO M, et al. Knowledgeon medical plantsand herbal medicines by Community health agentes of ljuí/RS. J. Res.: fundam care online, 2017; 9(1):57-63. Disponível em: http://www.seer.unirio.br/index.php/cuidadofundamental/article/view/4271/pdf_1

19. SILVA LAD, ALBIERO ALM. Programas de fitoterapia na atenção primária à saúde: existem experiências exitosas? Rev Bras. Farm, 2014; 95(3): 889-908.

20. SILVA MIG, et al. Utilização de fitoterápicos nas unidades básicas de atenção à saúde da família no município de Maracanaú (CE). Ver BrasFarmacogn, 2006; 16(4): 455-462.

21. SILVA TC, et al. Perfil de utilização de fitoterápicos em uma farmácia comunitária. $R$ Interd, 2018;11(3):61-66. Disponível em: https://revistainterdisciplinar.uninovafapi.edu.br/index.php/revinter/article/view/1447/pdf_33

22. SOUSA IV, et al. Coping with problems that impact on the health hof a socially vulnerable community from the resident's perspective. Cien Saude Colet, 2019; 24(5): 1647-1656.

23. TESSER CD, et al. Práticas Integrativas e Complementares na Atenção Primária à Saúde brasileira. Saúde Debate, 2018; 42(12): 174-188.

24. THIAGO SCS, TESSER CD. Percepção de médicos e enfermeiros da Estratégia de Saúde da Família sobre terapias complementares. Rev Saude Publica, 2011; 45(2):249- 257.

25. THIOLLENT M. Metodologia da pesquisa-ação. 16ª ed. São Paulo: Cortez; 2008.

26. WORLD HEALTH ORGANIZATION (WHO). Acupuncture: review andanalysisofreportsoncontrolledclinicaltrials. Geneva: WHO; 2002.

27. WORLD HEALTH ORGANIZATION (WHO). WHO Traditional Medicine Strategy 2002 - 2005.Geneva: WHO; 2002. 